\title{
Mortalité par maladie cardiovasculaire chez les \\ Premières nations au Canada, 1991-2001
}

\author{
M. Tjepkema, M.S.P. (1); R. Wilkins, M. Urb. (1,2); N. Goedhuis, B. Sc. (3); J. Pennock, M. Sc. (3)
}

Cet article a fait l'objet d'une évaluation par les pairs.

\section{Résumé}

Objectifs : Comparer les tendances en matière de mortalité par maladie cardiovasculaire chez les adultes appartenant aux Premières nations et chez les adultes non autochtones, par sexe, par quintile de suffisance du revenu et par niveau de scolarité.

Méthodologie : Un échantillon de 15 \% des répondants au recensement du Canada de 1991 âgés de 25 ans et plus avait été couplé précédemment avec 11 années de données sur la mortalité. Dans cette étude, on a considéré comme membres des Premières nations les personnes ayant déclaré une ascendance d'Indien d'Amérique du Nord, un statut d'Indien inscrit en vertu de la Loi sur les Indiens ou l'appartenance à bande indienne ou à une Première nation. La cohorte comprenait 62400 membres des Premières nations et 2624300 non-Autochtones.

Résultats : Le taux de mortalité normalisé selon l'âge par maladie cardiovasculaire était de $30 \%$ plus élevé chez les hommes des Premières nations et de $76 \%$ plus élevé chez les femmes des Premières nations que chez les personnes de même sexe de la cohorte non autochtone. Ces proportions représentent des excès de respectivement 58 et 71 décès pour 100000 personnes-années à risque chez les hommes et les femmes des Premières nations. Pour chaque quintile de suffisance du revenu (ajusté en fonction de la taille de la famille et de la région de résidence) et chaque niveau de scolarité, le risque de décéder d'une maladie cardiovasculaire était plus élevé chez les membres des Premières nations que chez les non-Autochtones.

Conclusion : Les membres des Premières nations ont présenté des taux plus élevés de mortalité par maladie cardiovasculaire que les Canadiens non autochtones dans chaque quintile de revenu et pour chaque niveau de scolarité. Le revenu et le niveau de scolarité ont expliqué $67 \%$ de la surmortalité chez les hommes des Premières nations et $25 \%$ chez les femmes.

Mots-clés : indigène, Indien inscrit, Indien non inscrit, Autochtone, revenu, niveau de scolarité

\section{Introduction}

Partout dans le monde, le fardeau de la maladie est disproportionné chez les peuples indigènes ${ }^{1,2}$. Historiquement, les maladies infectieuses étaient la principale cause du piètre état de santé des peuples autochtones ${ }^{3}$. Cependant, depuis les années 1960, on a observé chez les peuples autochtones une transition épidémiologique marquée par la hausse de la prévalence des maladies non transmissibles, telles que le diabète et les maladies cardiovasculaires, et par la baisse des maladies infectieuses ${ }^{4,5}$.

Les maladies cardiovasculaires sont une cause importante de décès prématurés, d'hospitalisations et d'invalidités ${ }^{6}$, et elles représentent un lourd fardeau pour le système de santé7. Des données d'enquête ont montré que la prévalence des maladies cardiovasculaires autodéclarées était plus élevée chez les membres des Premières nations vivant dans une réserve ${ }^{8}$, de même que chez les Autochtones (c'est-à-dire les membres des Premières nations, les Métis et les Inuits) vivant hors réserve, que chez les non-Autochtones ${ }^{9}$. Cela dit, les résultats fondés sur l'autodéclaration ne traduisent pas nécessairement l'écart véritable entre les membres des Premières nations et les autres Canadiens ${ }^{10}$. Un indicateur du fardeau de la maladie fondé sur les taux de mortalité serait plus fondamental et plus fiable pour suivre l'évolution des tendances. $\mathrm{Au}$ Canada, les enregistrements de décès ne contiennent généralement aucun identificateur des Premières nations, ce qui rend nécessaire un couplage de dossiers ou une approche fondée sur les régions. Les études dans lesquelles on a couplé des listes d'Indiens inscrits (membres des Premières nations inscrits en vertu de la Loi sur les Indiens du Canada) à des enregistrements de décès dans les statistiques de l'état civil ont montré que les taux de mortalité par maladie cardiovasculaire étaient plus élevés chez les Indiens inscrits que chez les autres Canadiens ${ }^{5,11,12}$. Toutefois, ces études ne prenaient pas en compte les personnes qui s'identifiaient comme membres des

Rattachement des auteurs :

1. Division de l'analyse de la santé, Statistique Canada, Ottawa (Ontario), Canada

2. Département d'épidémiologie et de médecine sociale, Université d'Ottawa, Ottawa (Ontario), Canada

3. Direction générale de la santé des Premières nations et des Inuits, Santé Canada, Ottawa (Ontario), Canada

Correspondance : Michael Tjepkema, Division de l’analyse de la santé, Statistique Canada, RHC-24Q, 100, promenade Tunney's Pasture, Ottawa (Ontario) K1A 0T6; tél. : 613-951-3896;

téléc. : 613-951-3959; courriel : michael.tjepkema@statcan.gc.ca 
Premières nations mais qui n'étaient pas inscrites en vertu de la Loi sur les Indiens (« Indiens non inscrits »), pas plus qu'elles ne fournissaient d'information sur le rôle éventuel du statut socioéconomique dans les écarts observés.

On estime que $80 \%$ des maladies cardiovasculaires prématurées peuvent être prévenues $^{7}$. Il devrait donc être possible de réduire de façon marquée le fardeau des maladies cardiovasculaires. Objectif " ambitieux, mais réaliste » pour 2020, la Stratégie canadienne de santé cardiovasculaire et Plan d'action (SCSC-PA) ${ }^{7}$ vise à diminuer le fardeau des maladies cardiovasculaires au sein des populations autochtones jusqu'à atteindre le même niveau que dans le reste de la population canadienne. Pour surveiller l'avancement vers cet objectif, il sera nécessaire de suivre les décès par maladie cardiovasculaire chez l'ensemble des Autochtones, à savoir les membres des Premières nations, les Métis et les Inuits. Cette étude vise à évaluer le fardeau de la mortalité par maladie cardiovasculaire chez les membres des Premières nations (Indiens inscrits ou non inscrits) et à comparer les taux à ceux observés chez les adultes non autochtones par sexe, par quintile de suffisance du revenu et par niveau de scolarité.

\section{Méthodologie}

\section{Sources des données}

Notre étude repose sur une analyse secondaire des données issues de l'Étude canadienne de suivi de la mortalité selon le recensement, 1991-20013. Pour être admissibles à la cohorte de départ, les personnes devaient être âgés de 25 ans ou plus et avoir rempli le questionnaire long du recensement de 1991, ce qui excluait celles vivant en établissement. Pour faire l'objet d'un suivi de la mortalité, les répondants au recensement visés par l'étude devaient d'abord être couplés à un fichier de noms crypté extrait à partir des données non financières des déclarations de revenu. Environ 80 \% des enregistrements du recensement visés par l'étude ( $n=2860244$ ) ont été appariés avec le fichier de noms. Un échantillon aléatoire de répondants ( $\mathrm{n}=125093)$ a ensuite été retiré pour réduire la taille de la cohorte finale ( $\mathrm{n}=2735152)$ à $15 \%$ de la population canadienne, comme le stipule le protocole relatif au couplage des enregistrements. Cette cohorte a été couplée à la Base canadienne de données sur la mortalité (du 4 juin 1991 au 31 décembre 2001) à l'aide de méthodes de couplage probabiliste fondées principalement sur les noms et les dates de naissance ${ }^{14,15}$. On a estimé que la détermination des décès était légèrement inférieure (95-96 \%) chez les membres de la cohorte des Premières nations que dans la cohorte prise dans son ensemble (97\%). D’autres détails sur la création et sur le contenu du fichier précédemment couplé sont donnés ailleurs ${ }^{13}$. L'étude a visé à comparer les tendances en matière de mortalité par maladie cardiovasculaire chez les adultes appartenant aux Premières nations et chez les adultes non autochtones, par sexe, par quintile de suffisance du revenu et par niveau de scolarité.

L'Étude canadienne de suivi de la mortalité selon le recensement a été approuvée par le Comité des politiques de Statistique Canada après la tenue de consultations avec le Comité de la confidentialité et des mesures législatives de Statistique Canada, la Division des services d'accès et de contrôle des données et le Commissaire à la protection de la vie privée du Canada.

\section{Définitions}

Pour l'étude, on a défini les membres des Premières nations comme les répondants au recensement ayant déclaré une ascendance d'Indien d'Amérique du Nord, un statut d'Indien inscrit en vertu de la Loi sur les Indiens du Canada ou l'appartenance à une bande indienne ou à une Première nation. Environ les trois quarts des membres de la cohorte des Premières nations satisfaisaient aux trois critères. Environ $9 \%$ des membres de la cohorte des Premières nations n'ont pas indiqué être Indiens inscrits.

La cohorte non autochtone était constituée de tous les répondants au recensement hormis ceux ayant indiqué une ascendance d'Indien d'Amérique du Nord, de Métis ou d'Inuit, un statut d'Indien inscrit ou l'appartenance à une bande indienne de l'Amérique du Nord ou à une Première nation. Les membres de la cohorte non définis comme membres des Premières nations ou comme non-Autochtones ont été exclus de l'analyse.

Une collectivité des Premières nations (ou réserve indienne) renvoie à un territoire mis de côté par le gouvernement fédéral pour l'utilisation et l'occupation par un groupe ou une bande d'Indiens.

Le niveau de scolarité a été classé en deux catégories : pas de diplôme d'études secondaires ou au moins un diplôme d'études secondaires (ou un certificat d'une école de métiers).

Les quintiles de population selon la suffisance du revenu ont été calculés comme suit. Tout d'abord, pour chaque famille économique ou personne seule, le revenu total avant impôt et après transferts de toutes provenances a été regroupé pour tous les membres de la famille, et le ratio entre le revenu total et le seuil de faible revenu de Statistique Canada pour la taille de la famille et le groupe de taille de la collectivité applicable a été calculé ${ }^{16}$. Ainsi, tous les membres d'une même famille se sont vu attribuer le même ratio, qui a été calculé pour toutes les personnes sauf celles vivant en établissement (donc pour toute la population visée par l'étude), y compris les personnes vivant dans des réserves indiennes. La population a ensuite été classée en fonction de ce ratio, et les quintiles de population ont été calculés pour chaque région métropolitaine de recensement, agglomération de recensement, ou pour l'ensemble de la population vivant en région rurale et petite ville dans une province ou un territoire donné. Le calcul des quintiles dans chaque région permet de tenir compte des différences régionales des coûts de logement, qui ne sont pas intégréees aux seuils de faible revenu. Pour les besoins de l'analyse, on a regroupé les quintiles 4 et 5 en raison du faible nombre de répondants des Premières nations appartenant à ces catégories.

\section{Techniques d'analyse}

On a calculé pour chaque membre de la cohorte les personnes-jours de suivi du début de l'étude (4 juin 1991) jusqu'à la date de décès, la date d'émigration (connue pour 1991 seulement) ou la fin de l'étude (31 décembre 2001). Pour chaque 
catégorie de maladie cardiovasculaire, on a établi des taux de mortalité selon l'âge et le sexe par tranche d'âge de 5 ans (à la date de référence), puis, en utilisant la structure démographique de la cohorte de l'ensemble des Autochtones (personnesannées à risque) comme population type (méthode de pondération interne), on a calculé des taux de mortalité normalisés selon l'âge (TMNA), selon le sexe et la sous-population. On a aussi calculé des rapports de taux (RT) et des différences de taux (DT) en comparant les TMNA des cohortes des Premières nations et ceux des non-Autochtones. Les différences de taux ont servi à mesurer la surmortalité. Des intervalles de confiance (IC) à $95 \%$ ont été établis pour les TMNA, les RT et les DT selon une distribution de Poisson.

Des rapports de risques proportionnels de Cox ont été utilisés pour calculer le risque de mortalité par maladie cardiovasculaire chez les membres des cohortes des Premières nations et des non-Autochtones selon le sexe, après des ajustements successifs en fonction de l'âge (en années), puis de l'âge et du niveau de scolarité (pas de diplôme d'études secondaires ou au moins un diplôme d'études secondaires) et enfin de l'âge et de la suffisance du revenu (quintiles 1, 2 et 3 par rapport aux quintiles 4 et 5 regroupés). Le modèle final était pleinement ajusté en fonction de l'âge, du niveau de scolarité et de la suffisance du revenu (de façon simultanée). Les différences dans les rapports de risques entre le modèle ajusté en fonction de l'âge et le modèle pleinement ajusté ont été interprétées comme des estimations de l'effet du niveau de scolarité et du revenu sur l'ampleur des écarts entre les adultes membres des Premières nations et les adultes non autochtones. La part de la surmortalité attribuable aux différences relevant du niveau de scolarité et du revenu a été calculée de la manière suivante : différence entre les rapports ajustés en fonction de l'âge et les rapports pleinement ajustés pour les Autochtones, divisée par le rapport ajusté en fonction de l'âge moins 1 .

La cause de décès avait préalablement été codée selon la Classification internationale des maladies, neuvième révision (CIM-9) de l'Organisation mondiale de la Santé dans le cas des décès survenus entre 1991 et 1999 et selon la Dixième révision (CIM-10) dans le cas des décès survenus en 2000 et en 2001. Les codes de la CIM ont été regroupés comme suit : toutes les maladies cardiovasculaires (CIM-9 : 390-459; CIM-10 : I00-I99), cardiopathie ischémique (CIM-9 : 410-414; CIM-10 : I20-I25), infarctus aigu du myocarde (CIM-9 : 410; CIM-10 : I21-I22), maladie vasculaire cérébrale (CIM-9) : 430-438; CIM-10 : I60-I69), accident vasculaire cérébral (CIM-9 : 430, 431, 434, 436; CIM-10 : I60, I61, I63, I64), insuffisance cardiaque congestive (CIM-9 : 428.0; CIM-10 : I50.0), cardiopathie inflammatoire (CIM-9 : 420-422, 425; CIM-10 : I30-I33, I38, I40, I42), cardite rhumatismale (CIM-9 : 390-398; CIM-10 : I01-I09) et cardiopathie hypertensive (CIM-9 : 401-405; CIM-10 : I10-I13).

\section{Résultats}

$\mathrm{Au}$ total, on a effectué un suivi de la mortalité chez 27300 hommes des Premières nations, 1307800 hommes non autochtones, 35100 femmes des Premières nations et 1316500 femmes non autochtones. Comparativement aux membres de la cohorte non autochtone, les membres de la cohorte des Premières nations étaient plus jeunes, avaient un niveau de scolarité moins élevé, avaient un revenu moindre et étaient plus nombreux à vivre dans l'Ouest canadien et dans le Nord (tableau 1).

Les décès par maladie cardiovasculaire représentaient $29 \%$ de tous les décès chez les hommes des Premières nations et $27 \%$ chez les femmes. La principale cause de mortalité par maladie cardiovasculaire était les cardiopathies ischémiques $(62 \%$ de tous les décès par maladie cardiovasculaire chez les hommes des Premières nations; $45 \%$ chez les femmes des Premières nations), suivies des maladies cardiovasculaires (14 \% chez les hommes des Premières nations; $25 \%$ chez les femmes des Premières nations). Les hommes des Premières nations présentaient un risque de décès par maladie cardiovasculaire de $30 \%$ plus élevé et les femmes de $76 \%$ plus élevé que les membres de la cohorte non autochtone. Cela équivaut à 58 décès supplémentaires pour 100000 personnes-années à risque chez les hommes des Premières nations et à 71 décès supplémentaires pour 100000 personnesannées à risque chez les femmes des Premières nations (tableau 2).

Le risque relatif de décès était particulièrement élevé chez les hommes et les femmes des Premières nations comparativement aux membres de la cohorte non autochtone en ce qui concerne les cardites rhumatismales (RT $=3,8$ et 2,9 respectivement), les insuffisances cardiaques congestives ( $\mathrm{RT}=2,2$ et 3,2 respectivement), les cardiopathies inflammatoires (RT $=1,7$ et 2,3 respectivement), les accidents vasculaires cérébraux ( $\mathrm{RT}=1,3$ et 2,0 respectivement) et les cardiopathies hypertensives (RT $=2,1$ chez les femmes des Premières nations) (tableau 2).

Les DT, une mesure du fardeau absolu, indiquent que les cardiopathies ischémiques étaient responsables de la majorité de la surmortalité par maladie cardiovasculaire (61 \% de la DT) chez les hommes des Premières nations, tandis que les cardiopathies ischémiques ( $36 \%$ de la DT) et les maladies vasculaires cérébrales (29 \% de la DT) étaient les principales causes de cette surmortalité chez les femmes des Premières nations (tableau 2).

Les TMNA concernant la mortalité par maladie cardiovasculaire étaient à leur plus haut chez les membres des Premières nations vivant dans la région de l'Atlantique (Nouveau-Brunswick, Île-du-Prince-Édouard, Nouvelle-Écosse, Terre-Neuve-et-Labrador) et au Manitoba et à leur plus bas chez ceux vivant au Québec et dans les territoires (Yukon, Territoires du Nord-Ouest, Nunavut) (tableau 3). Les TMNA relatifs aux maladies vasculaires cérébrales étaient semblables pour l'ensemble des membres des Premières nations, qu'ils vivent dans une réserve ou hors réserve (tableau 3 ).

Le risque relatif de décès par maladie cardiovasculaire dans la cohorte des Premières nations (comparativement aux membres de la cohorte non autochtone) était à son maximum dans les groupes d'âge les plus jeunes et diminuait avec l'âge (figure 1). Chez les membres des Premières nations âgés de 25 à 34 ans à la date de référence, le risque de décès par maladie 
TABLEAU 1

Caractéristiques des hommes et des femmes des Premières nations et des hommes et des femmes non autochtones, sujets ne vivant pas en établissement âgés de 25 ans ou plus à la date de référence, Canada, 1991

\begin{tabular}{|c|c|c|c|c|}
\hline & \multicolumn{2}{|c|}{$\begin{array}{c}\text { Membres des Premières } \\
\text { nations }\end{array}$} & \multicolumn{2}{|c|}{ Non-Autochtones } \\
\hline & Hommes & Femmes & Hommes & Femmes \\
\hline Nombre & 27300 & 35100 & 1307800 & 1316500 \\
\hline Pourcentage & 100 & 100 & 100 & 100 \\
\hline \multicolumn{5}{|l|}{ Tranche d'âge, ans (\%) } \\
\hline $25-34$ & 41 & 44 & 27 & 28 \\
\hline $35-44$ & 28 & 28 & 26 & 26 \\
\hline $45-54$ & 16 & 15 & 18 & 17 \\
\hline $55-64$ & 9 & 8 & 14 & 12 \\
\hline $65-74$ & 5 & 4 & 10 & 10 \\
\hline $75-84$ & 2 & 2 & 4 & 5 \\
\hline 85 et plus & $<1$ & $<1$ & 1 & 1 \\
\hline \multicolumn{5}{|l|}{ Province, région ou territoire de résidence (\%) } \\
\hline Canada atlantique ${ }^{a}$ & 5 & 5 & 8 & 8 \\
\hline Québec & 12 & 13 & 26 & 26 \\
\hline Ontario & 18 & 17 & 37 & 37 \\
\hline Manitoba & 18 & 16 & 4 & 4 \\
\hline Saskatchewan & 12 & 13 & 4 & 3 \\
\hline Alberta & 9 & 11 & 9 & 9 \\
\hline Colombie-Britannique & 20 & 19 & 12 & 12 \\
\hline Territoires $^{\mathrm{b}}$ & 6 & 5 & 1 & 0 \\
\hline \multicolumn{5}{|c|}{ Résidence dans une collectivité des Premières nations (\%) } \\
\hline Oui & 67 & 63 & 0 & 0 \\
\hline Non & 33 & 37 & 100 & 100 \\
\hline \multicolumn{5}{|l|}{ Niveau de scolarité (\%) } \\
\hline Pas de diplôme d'études secondaires & 59 & 55 & 34 & 34 \\
\hline Diplôme d'études secondaires & 33 & 29 & 38 & 35 \\
\hline Diplôme d'études post-secondaires & 7 & 13 & 13 & 19 \\
\hline Diplôme universitaire & 2 & 3 & 15 & 12 \\
\hline \multicolumn{5}{|l|}{ Quintile de suffisance du revenu (\%) } \\
\hline Quintile 1 - le moins élevé & 38 & 42 & 14 & 19 \\
\hline Quintile 2 & 26 & 25 & 19 & 19 \\
\hline Quintile 3 & 18 & 17 & 21 & 20 \\
\hline Quintile 4 & 12 & 11 & 23 & 20 \\
\hline Quintile 5- le plus élevé & 6 & 6 & 23 & 21 \\
\hline
\end{tabular}

Source : Étude canadienne de suivi de la mortalité selon le recensement, 1991 à 2001.

Remarque : Les dénombrements ont été arrondis à la centaine près.

${ }^{a}$ Nouveau-Brunswick, Île-du-Prince-Édouard, Nouvelle-Écosse et Terre-Neuve-et-Labrador.

${ }^{b}$ Yukon, Territoires du Nord-Ouest et Nunavut.

cardiovasculaire était $62 \%$ plus élevé chez les hommes et $217 \%$ plus élevé chez les femmes comparativement aux nonAutochtones du même sexe, alors que le risque relatif de décès par maladie cardiovasculaire était légèrement plus faible chez les hommes des Premières nations âgés de 75 ans et plus et semblable chez les femmes des Premières nations âgées de 85 ans et plus.
Les TMNA relatifs aux maladies cardiovasculaires étaient plus élevés chez les répondants n’ayant pas de diplôme d'études secondaires que chez ceux ayant au moins un diplôme d'études secondaires, tant dans la cohorte des Premières nations que dans la cohorte non autochtone (tableau 4). Des risques relatifs plus élevés (chez les membres des Premières nations comparativement aux non-Autochtones) étaient observables dans les deux catégories de niveau de scolarité et pour les deux sexes. En ce qui concerne les hommes des Premières nations comparativement aux hommes non autochtones, les RT et les DT étaient relativement plus élevés chez les détenteurs d'au moins un diplôme d'études secondaires. Quant aux femmes des Premières nations comparativement aux femmes non autochtones, les RT et les DT étaient plus élevés, quel que soit le niveau du scolarité (tableau 4).

Selon les quintiles de suffisance du revenu, les taux de mortalité par maladie cardiovasculaire se présentaient sous la forme d'un gradient en escalier (le quintile le plus faible ayant le taux de mortalité le plus élevé) tant dans la cohorte des Premières nations que dans la cohorte non autochtone (tableau 4). Le gradient de mortalité était plus prononcé chez les membres de la cohorte non autochtone que chez les membres de la cohorte des Premières nations. Des risques relatifs plus élevés (chez les membres des Premières nations comparativement aux non-Autochtones) étaient observables dans chaque tranche de revenu, les ratios les plus hauts ayant été observés dans le quintile de revenu le plus élevé (RT $=1,29$ chez les hommes des Premières nations; RT $=1,91$ chez les femmes des Premières nations).

Après ajustement en fonction du niveau de scolarité et de la suffisance du revenu, le risque de décès par maladie cardiovasculaire était plus élevé de $8 \%$ chez les hommes des Premières nations et de $50 \%$ chez les femmes des Premières nations (comparativement aux personnes du même sexe de la cohorte non autochtone) (tableau 5). Sur le plan des rapports de risques ajustés selon l'âge, le risque relatif de décès par maladie cardiovasculaire (après ajustement en fonction du niveau de scolarité et du revenu) était réduit de $67 \%$ (de 1,24 à 1,08) chez les hommes des Premières nations et de $25 \%$ (de 1,67 à 1,50) chez les femmes des Premières nations.

\section{Analyse}

Cette étude a été la première au Canada à évaluer les taux de mortalité par maladie cardiovasculaire chez les Premières nations 
TABLEAU 2

Ensemble des décès par maladie cardiovasculaire par sous-type, taux de mortalité normalisés selon l’âge pour 100000 personnes-années à risque, rapports des taux et différences des taux pour 100000 personnes-années à risque chez les hommes et les femmes des Premières nations comparativement aux hommes et aux femmes non autochtones, par tranche d'âge, sujets ne vivant pas en établissement âgés de 25 ans ou plus à la date de référence, Canada, 1991-2001

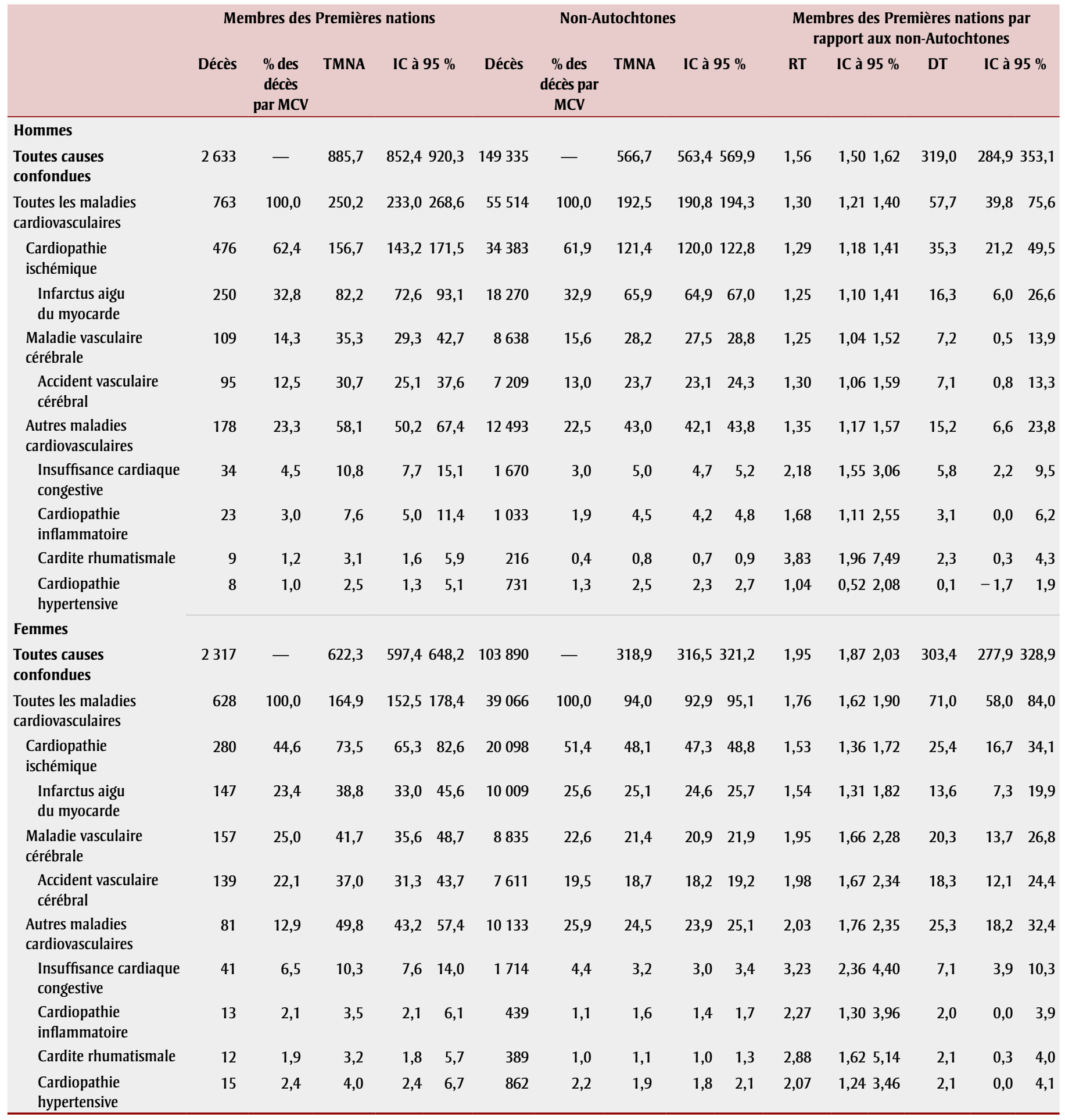

Source : Étude canadienne de suivi de la mortalité selon le recensement, 1991 à 2001.

Abréviations : DT, différence des taux; IC, intervalle de confiance; MCV, maladie cardiovasculaire; RT, rapport des taux; TMNA, taux de mortalité normalisés selon l'âge.

Remarque : La population type (personnes-années à risque) pour la normalisation selon l'âge est extraite de la répartition des Autochtones selon l'âge (tranches d'âge de 5 ans). 
TABLEAU 3

Ensemble des décès par maladie cardiovasculaire par sous-type, taux de mortalité normalisés selon l'âge pour 100000 personnes-années à risque, rapports des taux et différences des taux pour 100000 personnes-années à risque chez les hommes et les femmes des Premières nations comparativement aux hommes et aux femmes non autochtones, par région géographique, sujets ne vivant pas en établissement âgés de 25 ans ou plus à la date de référence, Canada, 1991-2001

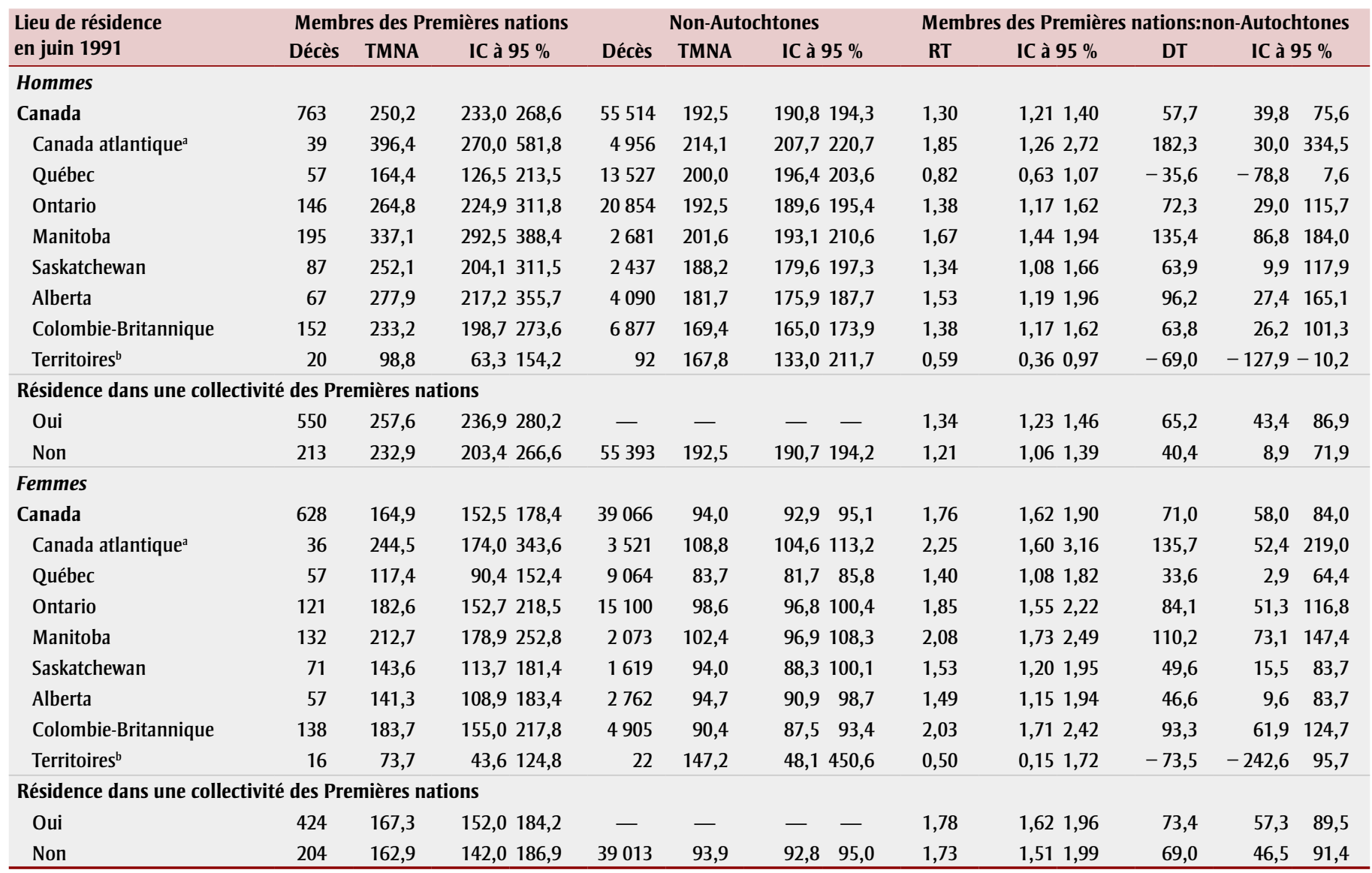

Source : Étude canadienne de suivi de la mortalité selon le recensement, 1991 à 2001.

Abréviations : DT, différence des taux; IC, intervalle de confiance; MCV, maladie cardiovasculaire; RT, rapport des taux; TMNA, taux de mortalité normalisés selon l'âge.

Remarque : La population type (personnes-années à risque) pour la normalisation selon l'âge est extraite de la répartition des Autochtones selon l'âge (tranches d'âge de 5 ans).

${ }^{\mathrm{a}}$ Nouveau-Brunswick, île-du-Prince-Édouard, Nouvelle-Écosse et Terre-Neuve-et-Labrador.

${ }^{b}$ Yukon, Territoires du Nord-Ouest et Nunavut.

FIGURE 1

Rapports des taux concernant la mortalité par MCV chez les membres de la cohorte des Premières nations comparativement aux membres de la cohorte non autochtone

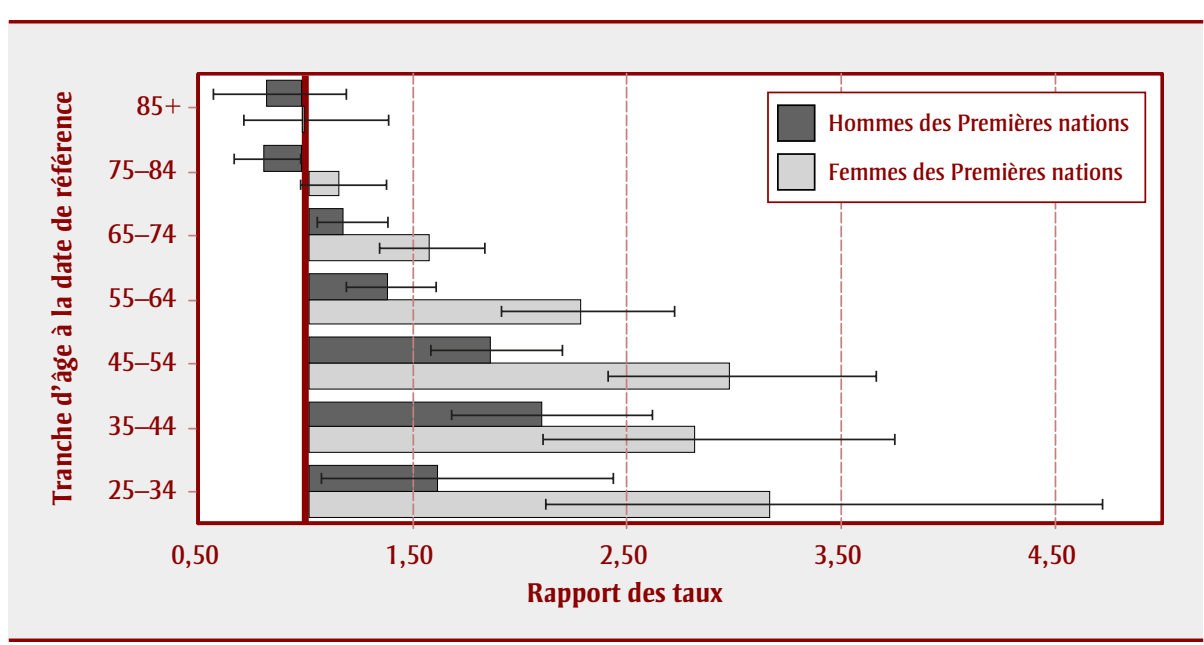

en fonction du niveau de scolarité et de la suffisance du revenu. Les résultats montrent que les adultes des Premières nations présentaient un risque accru de décès par maladie cardiovasculaire comparativement aux adultes non autochtones, tant dans l'ensemble que dans des sous-populations regroupées par niveau de scolarité et par revenu. L'étude incluait les membres des Premières nations qui n'étaient pas des Indiens inscrits en vertu de la Loi sur les Indiens du Canada (Indiens non inscrits) et incluait des données de toutes les provinces et tous les territoires du Canada.

Le fardeau des maladies cardiovasculaires augmente chez les Autochtones du Canada depuis plusieurs décennies ${ }^{5,7,17}$. Cependant, selon des données de la 
Colombie-Britannique, les TMNA relatifs aux maladies cardiovasculaires chez les Indiens inscrits ont diminué entre 1993 et 2006, quoiqu'ils soient demeurés plus élevés de $25 \%$ par rapport à ceux des autres habitants de la Colombie-Britannique ${ }^{12}$.

Des études ont révélé que les facteurs de risque classiques des maladies cardiovasculaires sont plus fréquents chez les membres des Premières nations que chez les non-Autochtones. Ils comprennent notamment un taux élevé de tabagisme $e^{8,9,18,19}$, l'hypertension artérielle ${ }^{8,9}$, l'obésité8,20, le diabète $^{21,22}$ et une mauvaise alimentation ${ }^{22-24}$.

Certains ont avancé que, en plus de ces facteurs de risque de maladie cardiovasculaire reconnus, des facteurs sociaux tels que le niveau de scolarité et le revenu étaient déterminants dans la survenue des maladies $^{25}$. Le niveau de scolarité et le revenu des membres des Premières nations sont inférieurs à ceux du reste de la population canadienne ${ }^{8,26}$. On a examiné la relation entre, d'une part, le niveau de scolarité et la suffisance du revenu et, d'autre part, les écarts sur le plan de la mortalité par maladie cardiovasculaire en comparant une cohorte de membres des

\section{TABLEAU 4}

Ensemble des décès par maladie cardiovasculaire, taux de mortalité normalisés selon l'âge pour 100000 personnes-années à risque, rapports des taux et différences des taux pour 100000 personnes-années à risque chez les hommes et les femmes des Premières nations comparativement aux hommes et aux femmes non autochtones, par indicateur socioéconomique, sujets ne vivant pas en établissement âgés de 25 ans ou plus à la date de référence, Canada, 1991-2001

\begin{tabular}{|c|c|c|c|c|c|c|c|c|c|c|}
\hline \multirow[t]{2}{*}{ Caractéristique évaluée en 1991} & \multicolumn{3}{|c|}{ Membres des Premières nations } & \multicolumn{3}{|c|}{ Non-Autochtones } & \multicolumn{4}{|c|}{$\begin{array}{c}\text { Membres des Premières nations par rapport aux } \\
\text { non-Autochtones }\end{array}$} \\
\hline & Décès & TMNA & IC à $95 \%$ & Décès & TMNA & IC à $95 \%$ & RT & IC à $95 \%$ & DT & IC à $95 \%$ \\
\hline \multicolumn{11}{|l|}{ Niveau de scolarité } \\
\hline \multicolumn{11}{|l|}{ Hommes } \\
\hline Pas de diplôme d'études secondaires & 606 & 256,7 & $236,3278,8$ & 33776 & 227,8 & $224,5231,1$ & 1,13 & $1,041,23$ & 28,9 & $7,4 \quad 50,4$ \\
\hline Diplôme d'études secondaires ou plus & 157 & 231,4 & $190,6281,0$ & 21738 & 166,4 & $164,2168,7$ & 1,39 & $1,141,69$ & 65,0 & $20,0110,0$ \\
\hline Pas de diplôme d'études secondaires & 530 & 176,3 & $161,3192,6$ & 25307 & 110,6 & $108,6112,7$ & 1,59 & $1,461,74$ & 65,7 & $49,981,4$ \\
\hline Diplôme d'études secondaires ou plus & 98 & 141,8 & $108,5185,4$ & 13759 & 79,7 & $78,3 \quad 81,1$ & 1,78 & $1,362,33$ & 62,1 & $24,1100,2$ \\
\hline \multicolumn{11}{|l|}{ Quintile de suffisance du revenu } \\
\hline \multicolumn{11}{|l|}{ Hommes } \\
\hline Quintile 1 - le moins élevé & 309 & 298,4 & $266,4334,3$ & 13053 & 273,1 & $267,3279,1$ & 1,09 & $0,971,23$ & 25,3 & $-9,1 \quad 59,7$ \\
\hline Quintile 1 - le moins élevé & 275 & 185,5 & $164,7209,0$ & 15918 & 127,7 & $124,5131,0$ & 1,45 & $1,291,64$ & 57,8 & $35,580,2$ \\
\hline Quintile 2 & 211 & 172,6 & $149,8198,8$ & 9346 & 98,6 & $96,0101,3$ & 1,75 & $1,512,02$ & 74,0 & $49,4 \quad 98,6$ \\
\hline Quintile 3 & 81 & 142,2 & $113,6178,1$ & 5559 & 88,9 & $86,4 \quad 91,5$ & 1,60 & $1,28 \quad 2,01$ & 53,3 & $21,285,4$ \\
\hline Quintiles 4,5 - les plus élevés & 61 & 146,7 & $112,1192,0$ & 8243 & 76,7 & $75,1 \quad 78,5$ & 1,91 & $1,462,50$ & 70,0 & $30,5109,4$ \\
\hline
\end{tabular}

Source : Étude canadienne de suivi de la mortalité selon le recensement, 1991 à 2001.

Abréviations : TMNA, taux de mortalité normalisés selon l'âge; IC, intervalle de confiance; MCV, maladie cardiovasculaire; DT, différence des taux; RT, rapport des taux.

Remarque : La population type (personnes-années à risque) pour la normalisation selon l'âge est extraite de la répartition des Autochtones selon l'âge (tranches d'âge de 5 ans).

TABLEAU 5

Rapports de risques de décès par maladie cardiovasculaire dans la cohorte des membres des Premières nations comparativement à la cohorte non autochtone, ajustés en fonction de certains indicateurs socioéconomiques, sujets ne vivant pas en établissement âgés de 25 ans ou plus à la date de référence, Canada, 1991-2001

\begin{tabular}{|c|c|c|c|c|c|c|}
\hline \multirow{3}{*}{$\begin{array}{l}\text { Ajusté en fonction de : } \\
\text { l'âge }\end{array}$} & \multicolumn{3}{|c|}{ Hommes } & \multicolumn{3}{|c|}{ Femmes } \\
\hline & \multirow{2}{*}{$\begin{array}{c}\begin{array}{c}\text { Rapport de } \\
\text { risques }\end{array} \\
1,24 \\
\end{array}$} & \multicolumn{2}{|c|}{ IC à $95 \%$} & \multirow{2}{*}{$\begin{array}{c}\begin{array}{c}\text { Rapport de } \\
\text { risques }\end{array} \\
1,67 \\
\end{array}$} & \multicolumn{2}{|c|}{ IC à 95 \% } \\
\hline & & 1,16 & 1,34 & & 1,54 & 1,80 \\
\hline l'âge + la suffisance du revenu & 1,13 & 1,05 & 1,21 & 1,58 & 1,46 & 1,71 \\
\hline l'âge + le niveau de scolarité + la suffisance du revenu & 1,08 & 1,00 & 1,16 & 1,50 & 1,39 & 1,63 \\
\hline
\end{tabular}

Source : Étude canadienne de suivi de la mortalité selon le recensement, 1991 à 2001.

Abréviation : IC, intervalle de confiance.

Remarque : Modèles ajustés en fonction de l'âge en années (en continu), du niveau de scolarité (détention d'au moins un diplôme d'études secondaires ou non) et du quintile de suffisance du revenu (1, 2 ou 3 vs $4+5$ regroupés). 
Premières nations et une cohorte de nonAutochtones. Les écarts étaient toujours présents après le calcul des taux de mortalité dans chaque catégorie de niveau de scolarité et de revenu, ce qui veut dire qu'ils ne peuvent pas être expliqués uniquement par ces facteurs. Dans des modèles de Cox ajustés en fonction à la fois du revenu et du niveau de scolarité, les rapports de risques étaient réduits de $67 \%$ chez les hommes et de $25 \%$ chez les femmes, indiquant que ces facteurs jouent un rôle important dans les écarts, mais qu'ils n'en sont pas entièrement responsables. Une étude portant sur l'impact du statut socioéconomique sur les inégalités dans l'état de santé auto-évalué et dans les maladies chroniques - recherche où étaient comparés des membres des Premières nations vivant hors réserve et d'autres Canadiens - a montré que la prise en compte de facteurs tels que le revenu et le niveau de scolarité diminuaient, mais n'éliminaient pas, ces écarts de santé ${ }^{27}$. Cette même étude a aussi montré que certains facteurs classiquement associés à la santé dans la population générale n'ont pas cet effet chez les Premières nations ${ }^{27}$. Par exemple, chez les adultes non autochtones, les hommes sont moins nombreux que les femmes à se déclarer en très bonne ou en excellente santé, tandis que chez les adultes des Premières nations, les hommes et les femmes sont aussi susceptibles les uns que les autres de se dire en très bonne ou en excellente santé. Bien que les résultats de notre étude aient montré que la mortalité par maladie cardiovasculaire est plus élevée chez les hommes que chez les femmes tant dans la cohorte des Premières nations que dans la cohorte non autochtone, la différence des taux entre les deux sexes était moins élevée dans la cohorte des Premières nations que dans la cohorte non autochtone. En termes de différences entre les sexes sur le plan de la mortalité par maladie cardiovasculaire, les femmes des Premières nations sont moins avantagées que ne le sont les femmes non autochtones par rapport aux hommes. D'autres études devraient être menées pour déterminer les causes de cette observation.

\section{Limites}

Les données de cette étude excluaient les personnes n'ayant pas rempli le questionnaire long du recensement de 1991, c'est-à-dire les résidents en établissement de soins de longue durée ou en centre pour personnes âgées, les détenus, de même que les personnes non dénombrées par le recensement (environ 3,4\% des résidents canadiens de tous âges). Les personnes non recensées sont susceptibles d'être des personnes jeunes, mobiles, à faible revenu, d'origine autochtone ${ }^{28}$, sans domicile fixe ou vivant dans une réserve indienne ${ }^{29}$. Par ailleurs, comme il était nécessaire d'obtenir des noms cryptés à partir des données des déclarations de revenu, seules les personnes déclarant un revenu ont pu faire l'objet d'un suivi de mortalité. Les taux de couplage avec le fichier de noms extrait à partir des données des déclarations de revenu étaient plus faibles pour les répondants au recensement appartenant aux Premières nations (54\%) que pour les répondants non autochtones $(77 \%)$. Le profil socioéconomique des membres de la cohorte des Premières nations était néanmoins semblable à celui de l'ensemble des membres des Premières nations ayant rempli le questionnaire long du recensement, ce qui milite en faveur de l'absence de biais important pendant le premier couplage (données non présentées).

Dans cette étude, on a défini les membres des Premières nations comme les répondants au recensement ayant déclaré une ascendance d'Indien d'Amérique du Nord, un statut d'Indien inscrit en vertu de la Loi sur les Indiens ou l'appartenance à une bande indienne ou à une Première nation, car le recensement de 1991 ne comportait pas de questions sur l'autoidentification à un groupe autochtone. Cette définition du membre des Premières nations excluait de nombreuses personnes d'origine mixte (autochtone et non autochtone) dont les caractéristiques au recensement (données non présentées) se rapprochaient davantage de celles des non-Autochtones que de celle des membres des Premières nations tels qu'ils ont été définis dans l'étude.

Notre étude portait sur la mortalité par maladie cardiovasculaire et non sur la morbidité. Quoique la mortalité représente l'aboutissement de l'évolution d'une maladie, l'étude ne constitue donc pas une évaluation du fardeau complet des maladies cardiovasculaires.
Le lieu de résidence, le niveau de scolarité et le revenu ont été évalués seulement à la date de référence (4 juin 1991) et ils ne correspondent donc pas nécessairement à la situation plus tard dans la période de suivi.

\section{Conclusion}

Le fardeau accru des maladies cardiovasculaires chez les peuples autochtones est de plus en plus reconnu au Canada ${ }^{4,7}$. Les résultats de cette étude montrent que la mortalité par maladie cardiovasculaire est plus élevée chez les membres des Premières nations en général et qu'elle est liée au niveau de scolarité et à la suffisance du revenu. Comme jusqu'à $80 \%$ des maladies cardiovasculaires prématurées sont considérées comme évitables, on devrait pouvoir réduire de façon marquée le fardeau des maladies cardiovasculaires chez les peuples autochtones du Canada? Les présents résultats montrent que la suffisance du revenu et le niveau de scolarité sont des facteurs qui jouent tous deux un rôle important dans les différences observées sur le plan des taux de mortalité par maladie cardiovasculaire entre les membres des Premières nations et les Canadiens non autochtones.

\section{Remerciements}

Cette étude a été financée par la Direction générale de la santé des Premières nations et des Inuits de Santé Canada. La création de la cohorte a été financée par l'Initiative sur la santé de la population canadienne, qui fait partie de l'Institut canadien d'information sur la santé. Les auteurs souhaitent également remercier Eric Guimond, d'Affaires autochtones et Développement du Nord Canada, pour avoir préalablement mis au point des définitions opérationnelles relatives aux Premières nations pour le recensement de 1991.

\section{Références}

1. Gracey M, King M. Indigenous health part 1: determinants and disease patterns. Lancet. 2009; 374(9683):65-75.

2. Initiative sur la santé de la population canadienne. Améliorer la santé des Canadiens. Ottawa (Ont.) : Institut canadien d'information sur la santé; 2004. 
3. Waldram JB, Herring DA, Young TK. Aboriginal health in Canada: historical, cultural, and epidemiological perspectives, $2^{e}$ éd. Toronto (Ont.): University of Toronto Press; 2006.

4. Reading J. The crisis of chronic disease among Aboriginal peoples: a challenge for public health, population health and social policy. Victoria (BC): Centre for Aboriginal Health Research, University of Victoria; 2009.

5. Trovato F. Canadian Indian mortality during the 1980s. Soc Biol. 2000; 47(1-2):135-45.

6. Lee DS, Chiu M, Manuel DG, Tu K, Wang X, Austin PC, et collab. Trends in risk factors for cardiovascular disease in Canada: temporal, socio-demographic and geographic factors. CMAJ. 2009;181(3-4): E55-E66.

7. Comité directeur de la SCSC-PA [Stratégie canadienne de santé cardiovasculaire et Plan d'action]. Pour un Canada au cœur qui bat. Ottawa (Ont.) : Stratégie canadienne de santé cardiovasculaire et Plan d'action; 2009 (consultation le 3 fév. 2010). PDF (298 Mo) téléchargeable à partir du lien : http:// www.chhs.ca/sites/default/files /Document \%20Links/Reports/French / CHHS \% 20 - \% 20Building \% 20a \% 20 Heart \% 20Healthy \% 20Canada \% 20- \% 20 FR\%20-\%20Fev\%202009.pdf

8. Équipe nationale de l'ERS. Enquête régionale longitudinale sur la santé des Premières Nations (ERS) 2002-2003 : Résultats de l'enquête sur les enfants, les jeunes et les adultes dans les communautés des Premières Nations. $2^{e}$ éd. rév. Ottawa (Ont.) : Assemblée des Premières Nations/Centre de gouvernance de l'information des premières nations; mars 2007 (consultation le 3 fév. 2010). PDF (en anglais) téléchargeable à partir du lien : www.rhs-ers.ca/sites/default/files /ENpdf/rhs2002-03reports/rhs2002-03technicalreport-afn.pdf

9. Lix LM, Bruce S, Sarkar J, Young TK. Facteurs de risque et problèmes de santé chroniques chez les Autochtones et les non-Autochtones. Rapports sur la santé. 2009; 20(4):1-10.

10. Anand SS, Yusuf S, Jacobs R, Davis AD, Yi Q, Gerstein $\mathrm{H}$, et al. Risk factors, atherosclerosis, and cardiovascular disease among Aboriginal people in Canada: the Study of Health Assessment and Risk Evaluation in Aboriginal Peoples (SHARE-AP). Lancet. 2001;58:1147-53.
11. Mao Y, Moloughney BW, Semenciw RM, Morrison HI. Indian reserve and registered Indian mortality in Canada. Can J Public Health. 1992;83(5):350-3.

12. British Columbia Provincial Health Officer. Pathways to health and healing: 2nd report on the health and well-being of Aboriginal people in British Columbia. Provincial Health Officer's Annual Report 2007. Victoria (BC): Ministry of Healthy Living and Sport; 2009.

13. Wilkins R, Tjepkema M, Mustard C, Choinière R. Étude canadienne de suivi de la mortalité selon le recensement, 1991 à 2001. Rapports sur la santé. 2008;19(3):27-48.

14. Fair ME. Generalized record linkage system: Statistics Canada's record linkage software. Austrian J Stat. 2004;33(1\&2):37-53.

15. Fair ME, Carpenter M, Aylwin H. Recherche sur la santé : catalogue des projets sur l'hygiène du travail et de l'environnement : 1978 à 2005. Ottawa (Ont.) : Statistique Canada; 2006 (Statistique Canada, $n^{\circ} 82-581$-XIF au catalogue).

16. Statistique Canada. Dictionnaire du recensement de 1991. Ottawa (Ont.) : Statistique Canada; 1992 (Statistique Canada, $n^{\circ}$ 92-301F au catalogue).

17. Shah BR, Hux JE, Zinman B. Increasing rates of ischemic heart disease in the native population of Ontario, Canada. Arch Intern Med. 2000;160(12):1862-6.

18. Anand SS, Razak F, Davis AD, Jacobs R, Vuksan V, Teo K, et al. Social disadvantage and cardiovascular disease: development of an index and analysis of age, sex, and ethnicity effects. Int $\mathrm{J}$ Epidemiol. 2006;35(5):1239-45.

19. Retnakaran R, Hanley AJ, Connelly PW, Harris SB, Zinman B. Cigarette smoking and cardiovascular risk factors among Aboriginal Canadian youths. CMAJ. 2005;173(8):885-9.

20. Katzmarzyk P. Obesity and physical activity among Aboriginal Canadians. Obesity. 2008;16(1):184-90.

21. Johnson JA, Vermeulen SU, Toth EL, Hemmelgarn BR, Ralph-Campbell K, Hugel G, et al. Increasing incidence and prevalence of diabetes among the Status Aboriginal population in urban and rural Alberta, 1995-2006. Can J Public Health. 2009;100(3):231-6.
22. Dyck R, Osgood N, Lin TH, Hsiang T, Gao A, Stang MR. Epidemiology of diabetes mellitus among First Nations and non-First Nations adults. CMAJ. 2010;182(3):249-56.

23. Dewailly E, Blanchet C, Gingras S, Lemieux S, Holub BJ. Cardiovascular disease risk factors and n-3 fatty acid status in the adult population of James Bay Cree. Am J Clin Nutr. 2002;76(1):85-92.

24. Garriguet D. L'obésité et les habitudes alimentaires de la population autochtone. Rapports sur la santé. 2008;19(1):1-17.

25. Link BG, Phelan J. Social conditions as fundamental causes of disease. J Health Soc Behav. 1995;35:80-94.

26. Statistique Canada. Peuples autochtones du Canada en 2006 : Inuits, Métis et Premières nations, Recensement de 2006. Ottawa (Ont.) : Statistique Canada; 2008 (Statistique Canada, $n^{\circ}$ 97-558-XIF au catalogue).

27. Garner R, Carrière G, Sanmartin C, équipe de recherche de l'Initiative sur les données longitudinales administratives et sur la santé. La santé des adultes chez les Premières Nations vivant hors réserve, les Inuits, et les Métis au Canada : l'incidence du statut socioéconomique sur les inégalités en matière de santé. Ottawa (Ont.) : Statistique Canada; 2010 (Série de documents de travail de la recherché sur la santé, $n^{\circ} 4$ ) (Statistique Canada, $\mathrm{n}^{0}$ 82-622-X au catalogue $\left.-n^{\circ} 004\right)$.

28. Statistique Canada. Couverture. Rapports techniques du recensement de 1991, Série des produits de référence. Ottawa (Ont.) : ministre de l'Industrie, des Sciences et de la Technologie; 1994 (Statistique Canada, $\mathrm{n}^{\circ}$ 92-341F au catalogue).

29. Statistique Canada. Enquête auprès des peuples autochtones de 1991 : fichier de microdonnées des adultes : guide de l'utilisateur. Ottawa (Ont.) : Statistique Canada; 1995. 